\title{
La jouissance féminine et le maternel en questions
}

\section{Feminine jouissance and the maternal in question}

Jessica Choukroun-Schenowitz*1

L'auteur se propose de confronter les deux champs contigus du féminin et du maternel à partir des considérations freudiennes sur le "continent noir" qu'est la féminité et de la notion de jouissance féminine déployée par Jacques Lacan.

A la lumière de la psychopathologie clinique psychanalytique, cet article veut mettre en évidence que si l'Edipe ne fait pas La femme, le fait du ravage qui en est sa conséquence, envahit aussi bien le féminin que le maternel. En effet, la part de jouissance opaque de la mère, soit le féminin dans la mère, contamine la féminité tout comme la maternité d'une femme, tel qu'en témoigne le rapport de ravage qui peut exister entre une fille et sa mère.

Il s'agira ainsi de pointer que, pas plus que le devenir femme, le devenir mère n'est donné d'emblée mais qu'il se construit du rapport au langage, au fantasme, à la jouissance et à l'Autre en la figure de l'amour maternel, pour chaque une.

Mots clés: Féminin, maternel, jouissance, ravage

${ }^{* 1}$ Université Côte d'Azur - Campus Carlone (Nice, France). 


\section{ARTIGOS}

\section{Pour ouvrir la question}

"La moindre conversation est là pour vous démontrer que l'amour de la mère est la cause de tout" disait Lacan (1961-62).

L'amour de la mère est en effet ce que la praxis analytique peut situer en lieu et place de l'instinct maternel parce que le langage est ce qui vient dénaturer l'animal humain.

Défini par Lacan (1957-58) comme le don de ce qu'on n'a pas (p. 351), l'amour convoque inévitablement la dimension du manque et de la castration chez l'aimant. Quand il se conjugue au féminin, l'amour peut rimer avec le ravage, ${ }^{1}$ lequel se déploie comme une conséquence de l'impossible liquidation de l'Edipe féminin. Que l'Edipe ne fasse pas La femme, que le symbolique soit inapte à dire ce que le féminin renferme d'énigmatique, est précisément ce que la notion de ravage recouvre.

Depuis Freud (1933/1984) qui assimilait la maternité à la féminité "normale" tout en repérant néanmoins et sans duperie les énigmes persistantes du continent noir, nous ne pouvons que tâcher de différencier le féminin du maternel et affirmer qu'une femme n'est pas qu'une mère et qu'une mère peut ne pas "être femme".

Et pourtant, cette dichotomie relève-t-elle vraiment de l'évidence? En quoi la mère et la femme restent-elles indissolublement liées? Comment le "féminin dans la mère"2 vient-il contaminer l'être femme mais aussi bien l'être mère d'une femme?

Reprenons pas à pas afin de déployer la question.

${ }^{1}$ Lacan le définit comme "le rapport d'une femme à sa mère, d'où elle semble bien attendre comme femme plus de subsistance que de son père, lequel arrive second dans ce ravage", "L'étourdit", Scilicet 4, Paris, Le Seuil, 1973.

${ }^{2}$ Cf. Choukroun-Schenowitz, J. "Le féminin dans la mère", in www.lacan-université.fr et "L'ombre de la mère". Communication aux 44èmes Journées de l'ECF (Palais des Congrès, Paris, 15 et 16 novembre 2014) que le présent article prolonge et approfondit. 


\section{L'Edipe ne fait pas La femme}

"Si vous voulez en savoir plus sur la féminité, interrogez vos propres expériences de la vie, ou adressez-vous aux poètes ou bien attendez que la science puisse vous donner des renseignements plus approfondis et plus cohérents" (Freud,1933/1984, p. 181).

Tel est ce que Freud finit par énoncer en 1933 laissant entendre combien le désir féminin demeure un mystère. Selon E. Solano-Suarez (2003, pp. 31-45), il s'agit là d'une impasse théorique: en érigeant le désir féminin en mystère, Freud soumet la sexualité féminine au régime de l'impuissance phallique, confondant du même coup féminité et hystérie.

C'est dire que la question féminine demeure, de façon insuffisante, articulée à celle du phallus par le biais du manque de pénis dont la femme ne se guérit finalement jamais.

En effet, Freud a défini la femme comme une version de l'homme, soit à partir de la logique binaire du phallus telle qu'elle prévaut dans l'inconscient.

De 1908 à 1933, l'ensemble des écrits freudiens chemine vers cette tentative de définir le féminin. D'abord, c'est à partir de la bipartition fort/ faible et actif/passif que vient s'inscrire la différence homme/femme pour l'inconscient (Freud, 1907/1997).

Puis, Freud (1907/1997) insistera sur le "besoin" spécifique à la femme qui ne la fait "pas tendre à aimer mais à être aimée" (p. 94), et affirmera que la voie qui mène une femme au "plein amour d'objet" réside dans la mise au monde de son enfant. De sa progéniture, la femme tentera d'obtenir un "dédommagement" même "tardif" dans ce que sa maternité peut lui apporter.

La difficulté sur laquelle bute le père de la psychanalyse découle d'un fait: le primat du phallus (Freud, 1907/1997, pp. 115-116). Au stade de l'organisation génitale infantile, l'opposition actif/passif laisse place à l'opposition: organe génital masculin ou châtré. S'il y a donc bien un masculin pour l'inconscient, il n'y a pas de féminin nous dit Freud.

Aussi, la disparition du complexe d'CEdipe s'effectuera par un désinvestissement du père au profit d'une identification, sous la menace de castration, chez le garçon, faisant apparaître le surmoi comme "héritier" du dit complexe. Mais pour la fille, pointera Freud, le "matériel devient — d'une façon incompréhensible - beaucoup plus obscur et lacunaire". Si celle-ci a un complexe d'œdipe, un surmoi, un temps de latence, une organisation phallique et un complexe de castration, 'le renoncement au pénis n'est pas supporté sans 
une tentative de compensation". Et de noter que "l'anatomie, c'est le destin", Freud ébauche le complexe de masculinité de la femme.

Aussi, le complexe d'CEdipe de la fille ne va rarement, repère Freud (1907/1997), “au-delà de la substitution à la mère et de la position féminine à l'égard du père" (p. 122). C'est ainsi que le glissement s'opère pour la fille le long d'une équation symbolique que nous connaissons (phallus = enfant) avec ses conséquences sur la position féminine.

En 1925, Freud affirme que le complexe d'œdipe de la petite fille recèle un problème de plus que celui du garçon et met en évidence que la question féminine semble davantage s'attacher à la différence des sexes qu'à l'origine des enfants. Au regard du complexe de castration, la fille entre ainsi dans l'Edipe "victime" de son "envie du pénis", alors que le garçon en sort. Cette dissymétrie freudienne sera maintenue chez Lacan qui, réaffirmant le phallocentrisme de l'inconscient, dira que: "La Femme n'existe pas" (Lacan, 1970-71). Il avait déjà précisé qu'il "n'y a pas de symbolisation du sexe de la femme comme tel" (Lacan, 1955-56/1981, p. 198).

Ainsi, à partir du complexe de castration qui signe pour la fille l'entrée dans l'Edipe, il reste à celle-ci, entre autres possibilités évoquées par Freud, celle de parvenir à une transformation du Penisneid en Peniswunsch (désir et non plus envie de pénis) avec le désir d'enfant du père qui s'en suit: c'est la féminité normale.

Néanmoins, cette "envie du pénis" laissera tout de même "des traces indélébiles dans le développement de la femme et la formation de son caractère en ceci qu'elle n'est pas surmontée sans une lourde dépense psychique, dans le cas le plus favorable" (Freud, 1933/1984). Et d'énumérer les diverses conséquences psychiques de l'envie du pénis chez la femme, Freud aborde le lien à la mère et précisément "le relâchement de la relation tendre à la mère en tant qu'objet", celle-ci étant rendue responsable d'avoir "lancé l'enfant dans la vie avec un équipement aussi insuffisant". Mais encore, il soutient en 1931 que c'est "la rancune contre l'empêchement de l'activité sexuelle libre qui joue un grand rôle dans la séparation d'avec la mère" (Freud, 1907/1997, pp. 130-146). Et si grâce à cette fameuse équation symbolique, "la petite fille tourne en femme", poursuit-il, en prenant son père comme objet d'amour, le sort de celle-ci n'en est pas réglé pour autant puisque le complexe de masculinité réapparaîtra au moment où la fille devra abandonner son lien au père, précisément en raison d'une identification à celui-ci.

Freud conclut donc sur le défaut d'un "motif élucidant la destructionaléatoire, insistons-nous — du complexe d'Edipe" chez la fille avant de 
se pencher sur l'importance de la préhistoire de l'Edipe, notamment en articulant l'envie du pénis à la haine que la fille éprouve envers sa mère.

La question qui anime Freud est alors de savoir "comment, quand et pourquoi" la petite fille se détache de sa mère (Freud, 1907/1997, pp. 139-144). Car, en effet, et de ce point précis s'ouvre la plage de ce que nous nommons après Lacan le ravage, Freud s'aperçoit qu' "il fallait admettre la possibilité qu'un certain nombre d'êtres féminins restent attachés à leur lien originaire avec la mère et ne parviennent jamais à le détourner véritablement sur l'homme".

Force est d'ailleurs de constater la difficulté dont témoigne Freud alors qu'il explique avoir tenté de "saisir analytiquement" tout ce qui touche au domaine de ce premier lien à la mère. Néanmoins, il en fera le berceau de l'hystérie, le germe de la paranoïa ultérieure de la femme qu'il assimile à une "angoisse surprenante mais que l'on trouve régulièrement". Mais plus encore, il affirme que "cette angoisse correspond à une hostilité envers la mère".

C'est ainsi la phase préœdipienne de lien exclusif à la mère qui retiendra l'attention de Freud référant dès lors cette hostilité à une conséquence non pas de la rivalité du complexe d'œdipe, mais bien "au contraire de la phase précédente" qui "n'a été que renforcée et exploitée dans la situation œdipienne".

Malgré l'équivalence phallus = enfant, Freud conclut toujours sur une "liquidation provisoire", une "position de repos" quant à l'œdipe féminin, entérinant son imparfaite abolition. De cet éclairage-là, il fait consister le roc de la castration en un refus de la féminité, le Penisneid se révélant comme un écueil dans l'analyse des sujets féminins.

C'est que le complexe d'œdipe de la petite fille recèle un problème de plus que celui du garçon. Le passage de la fille à la femme implique, en théorie, un changement d'objet, un changement d'identification, ou encore de zone génitale.

Freud fait ainsi de l'Edipe une "formation secondaire" (Freud, 1907/1997, p. 126) et affirme que le noyau de la névrose, s’il est œdipien pour le garçon, est plutôt préœdipien pour la fille.

\section{Le féminin et le maternel ou les deux faces du ravage}

Si la question du devenir femme demeure une énigme, qu'elle est insuffisamment recouverte dans cette tentative de faire équivaloir la maternité à la féminité "normale", c'est sans doute parce que, de ce féminin, rien n'existe pour l'inconscient. 
Le fait du ravage recouvre alors cette zone "pleine de lacunes et d'ombres" (Freud, 1907/1997, p. 122) que Freud, dans sa recherche de ce qui spécifie l'être femme, ne parvint pas à éclairer suffisamment.

Mais il eut tout de même une intuition de cette distinction alors qu'il énonça la division de la femme: si l'homme n'a qu'une seule zone génitale prédominante, "la femme en possède deux, le vagin qui est proprement féminin et le clitoris analogue au membre viril", divisant sa vie sexuelle en deux phases, nous dit-il, dont "seule la seconde est spécifiquement féminine" (Freud, 1907/1997, p. 142).

Freud annonçant ainsi ce supplément spécifique au féminin, Lacan parviendra à différencier la part des êtres parlants se rangeant sous le signifiant "femme" à partir du mode de jouissance et non plus de l'anatomie.

Le sujet féminin s'il se formule à partir de la signification phallique est aussi le sujet d'une jouissance Autre, jouissance "supplémentaire", féminine, qui va au-delà du phallus.

C'est donc au-delà de l'Edipe et au-delà du père, qu'il s'agissait d'aller chercher cet Autre sexe parce que "pour l'inconscient, la femme n'entre en jeu que quoad matrem" (Lacan, 1972-73, p. 30), c'est-à-dire qu'elle n'existe que comme mère.

A quoi l'enfant a-t-il alors affaire dans le rapport à sa mère? Et comment rendre compte de l'importance de cette phase pré-oedipienne si conséquente dans le devenir femme?

L'impossible liquidation de l'Edipe chez la fille annonce bon nombre d'impasses témoignant de l'identification problématique du sujet féminin. Que le père ne constitue pas une issue favorable à l'Edipe pour la fille intensifie la fixation, le retour à ses premières amours: la relation à la mère.

S'actualisant dans le rapport d'une fille à sa mère, le ravage s'illustre lorsque la mère demeure l'unique partenaire de la fille, barrant ainsi l'accès à la féminité et endommageant du même coup la maternité.

La passion pour la mère et la demande infantile à son endroit maintiennent la fille en position d'objet, avec les revendications qui en découlent, aussi nombreux que soient les partenaires qui se déclinent sur ce premier modèle. Freud lui-même repérait combien les femmes pouvaient reprendre les armes contre leur mari après avoir porté leurs griefs liés à leur manque phallique auprès de leur mère...

En sortant la femme de son équivalence exclusive à la mère, Lacan a mis en avant cette division entre mère et femme et fait valoir le féminin sous le signe d'un "plus" et non d'un moins, d'un manque. 
Pourtant, lorsque l'on s'interroge sur l'une ou l'autre de ces positions, il apparaît difficile de séparer simplement ces deux champs contigus du féminin et du maternel et c'est souvent le couple mère/femme qui est convoqué, que la mère soit ainsi pensée en regard de la femme, ou la femme en regard de la mère.

Nous pouvons alors distinguer deux positions qui rendent compte de cette division "pas-toute" entre mère et femme, pouvant se résumer dans les formulations suivantes: "Etre mère avant tout" ou "être toute mère" et "être femme avant tout" ou "être plus femme que mère"; formulations que nous entendons régulièrement dans la clinique des sujets féminins. A partir de ces positions, nous pouvons alors repérer deux versants du ravage.

L'un est plutôt articulé à la dimension phallique: nous y repérerons l'exclusivité, la demande d'amour, d'identité, de supplément d'être que nous observons chez ces sujets qui veulent demeurer l'objet unique de l'Autre maternel jusqu'au ravage. Ce qui par ailleurs pourra se répéter, souvent, dans leur propre maternité où elles continueront à demander, y compris à leur fille, ce qui était demandé à la mère.

L'autre est plutôt articulé à l'Autre jouissance, féminine, où la symbolisation ne cesse pas de faire défaut. Là, le type de rencontre de 576 l'enfant avec cette dimension de la jouissance énigmatique, folle, excessive de la femme dans la mère fait trauma et marque l'existence du sujet; cela sans doute parce que "dans le rapport sexuel, la femme n'entre en fonction" que comme mère (Lacan, 1972-73, p. 36), soit que dans l'inconscient, la femme n'apparaît que comme mère.

La figure connue et extrême que Lacan amène pour dire ce que serait la vraie femme, c'est Médée. La jouissance féminine, c'est ce champ qui relève du hors langage, de l'illimité, du corps et non plus seulement de l'organe phallique. Ici, la loi du père peine à faire le poids face au caprice de la mère et le reproche qui revient dans la bouche des sujets est celui d'avoir eu une mère "plus femme que mère".

Ce que montre ainsi le ravage, c'est la façon dont chacune peine à traiter l'énigme de la jouissance maternelle, son caprice de femme, cet endroit où elle n'est pas que mère, toute mère. Subissant l'impossible transmission d'un "être femme" par la mère, chaque une demande alors encore davantage de cette subsistance, de cet amour qui pourront lui décerner un être, la rendre consistante et par là, chaque une continue de s'adresser à une mère toute puissante, qu'il n'est d'ailleurs pas toujours possible de haïr. Et cette dissymétrie entre l'Edipe masculin et l'Edipe féminin rend sans doute encore plus effective la puissance de cette mère réelle. 


\section{ARTIGOS}

Cette "toute mère" qui fait flamber le ravage a donc pour corollaire un refus de la féminité au profit d'une fixation à la mère, une mère qui "convoque soit à la fusion impossible soit à la persécution" précise M. H. Brousse (2002). Les femmes et les mères en analyse témoignent donc du fait que barrer cet "Autre increvable" n'est pas tâche aisée et que le "pas-tout" ne consent pas toujours à faire "sans le ravage" (Marret-Maleval, 2010). Parce que l'amour et le ravage, côté femme, peuvent être les deux faces d'une même pièce (Miller, 1999, p. 15), qu'ils sont tous deux une réponse au non-rapport sexuel. Plus précisément, le ravage met l'amour à l'épreuve dans cette tentative de faire exister l'Autre, un Autre consistant, au lieu où il s'agirait plutôt pour une femme de s'éprouver elle-même comme Autre, assumant du même son corps et sa jouissance de femme.

\section{Anabelle ou l'ombre de la mère}

\section{D'abord, l'angoisse envahissante:}

Anabelle vient consulter précisément au moment où elle se découvre enceinte, à vingt-cinq ans, d'un homme auquel elle est très attachée malgré la violence de leur relation.

La situation est complexe et l'histoire est lourde. Aussi, l'angoisse, déjà présente, s'intensifie avec cette nouvelle grossesse qui sera menée à terme parce "devenir mère était une nécessité". En effet, si Anabelle a subi plusieurs interruptions de grossesse la rendant honteuse dans le passé, cette grossesse ne fait pas question pour elle puisqu'elle se présente sous le jour de la nécessité.

Néanmoins, la jeune femme craint de "s'apercevoir qu'elle ne supporte pas d'être enceinte" et plus, elle redoute d'être "comme sa mère". Et pour cause, sa mère, ayant contracté une maladie mortelle, est décédée dans des conditions dramatiques alors qu'Anabelle avait seize ans. Celle-ci aurait "commencé à avoir des problèmes psychiatriques" au moment de sa naissance.

Aussi, les conflits parentaux éclatent rapidement et la mère, versant dans une paranoïa, décide de se séparer de son mari durant alors qu'Anabelle est encore nourrisson. Le père, d'après les dires de la jeune femme, exerce fort difficilement sa fonction. Il aime beaucoup les enfants, certes, mais il ne sait pas vraiment les élever, déplore-t-elle.

C'est une tante maternelle, très présente depuis l'enfance d'Anabelle, qui l'élèvera bien que le père tente de "récupérer" sa fille. 
Les conflits entre les familles paternelle et maternelle sont nombreux, et Anabelle reprend à son compte diverses accusations faisant de son père le "conard" dont tout est la faute alors qu'elle revendique auprès de lui amour et reconnaissance. D'ailleurs, elle témoigne rapidement de l'existence d'une rivalité avec sa demi-sœur, fille d'une seconde union du père, qui "a donné un enfant au père la première".

Le diagnostic n'est pas aisé tant l'angoisse est présente, désorganisant quelque peu la pensée de la jeune femme. Et, il faudra du temps pour que celle-ci parvienne à sortir quelque peu de la confusion qui l'envahit.

Comment être mère quand la figure du ravage a pris les couleurs de la mort? L'être mère d'une femme peut-il se passer d'une identification à sa propre mère? C'est notre première question, et de fait, celle qui a présidé à l'arrivée en consultation d'Anabelle.

Le début de nos rencontres sera ainsi marqué par des absences et des retards aux rendez-vous. L'angoisse est au premier plan et Anabelle est débordée par les événements de sa vie et particulièrement par ses difficultés de couple au sein duquel la violence est de mise.

Le souci est d'emblée important face à cette patiente qu'il conviendra de 578 soutenir dans ce début de travail mais aussi de "rendre responsable" et non plus victime de son compagnon.

Que suis-je pour l'Autre... ou le ravage à l'œuvre:

Il faudra faire déconsister cette identification à la mère chez cette jeune femme qui, sidérée par l'énigme du décès maternel, commémore toutes les dates "anniversaires" de son histoire chargées symboliquement, par des effondrements. Les idées de suicide apparaissent chez la patiente à chaque moment où la question de ce qu'elle est pour l'autre est au premier plan: ce qu'elle a été pour sa mère d'abord, ce qu'elle est pour son père à qui elle n'a pas donné un enfant avant sa sœur, ce qu'elle est pour son compagnon qui la violente et qui ne voit pas sa souffrance. Et, ce qu'elle est dans le transfert parce qu'elle n'est pas reçue assez longtemps, pense-t-elle. Il s'agira de l'interpréter pour elle et de la rappeler à chaque fois qu'elle tente, parce qu'elle se sent trop mal, de se défiler et de manquer ses séances. Il conviendra de lui dire qu'elle est attendue de pied ferme pour tenter de l'accrocher à sa parole.

Le travail sera alors de plus en plus régulier mais difficile.

La violence avec son compagnon est en effet très fréquente et inquiétante alors que la jeune femme est enceinte et elle se poursuit en présence de 
l'enfant après sa naissance. Un jour, Anabelle dira qu'elle a pensé pouvoir mourir sous les coups de son compagnon.

Nous lui demandons alors de prendre soin d'elle et de sa fille.

\section{Le dégagement d'une question à traiter:}

Anabelle parviendra alors à préciser ce qu'elle nomme "l'héritage" dont elle "ne peut se défaire": ce "sentiment permanent d'être nulle, ratée, moins bien que les autres" qu'elle pense partager avec sa mère et qui serait la cause du décès de celle-ci. Mais une autre version suivra: "si elle est partie c'est que je n'ai pas été assez bien", ou encore: "ma mère est partie parce que j'ai rencontré quelqu'un et que je me suis éloignée d'elle". La veille de ce décès, Anabelle avait rejeté sa mère suite à un violent conflit entre elles.

Entendre cette culpabilité lui permettra de se décaler quelque peu de cette position d'être la cause des maux de l'autre et d'entamer un décollement de l'identification à la mère.

Ayant repéré son besoin de s'entourer de "gens qui ont des problèmes", Anabelle qui dit "subir le désir de l'autre", met en série ses relations d'objet en soulignant cette "emprise" et cette impression de "se faire bouffer": par sa mère, par son compagnon, par sa tante mais aussi à présent par sa fille avec laquelle les difficultés sont grandes.

Avec cette naissance et le sentiment de responsabilité qui en découle, la jeune femme mettra petit à petit tout en œuvre pour quitter ce partenaire même si la séparation sera difficile et couteuse.

Mère, pas-toute seule:

Parvenue à quitter son compagnon, Anabelle va vivre chez sa tante maternelle car être seule avec sa fille ne lui est pas supportable et lui semble impossible. Sa maternité est vécue dans l'angoisse et elle a besoin d'un tiers entre elle et sa fille.

Mais vivre chez sa tante la renvoie à s'identifier encore à sa mère qui a fait de même avec elle, bébé. Tout en revendiquant sa place de mère, elle en est à questionner sa propre violence et sa difficulté à faire seule avec sa fille alors qu'elle tente de se séparer de cette tante, figure maternelle toutepuissante dans cette constellation de pères absents, qui l'empêche d'occuper sa place.

Le soutien qu'elle trouve dans son travail thérapeutique devenu à présent régulier est important. Elle a consenti à dire sa honte, sa culpabilité mais aussi sa violence à l'égard de cet objet sans doute trop réel qu'est son enfant. Elle 
a également accepté d'aller consulter pour sa fille suivie depuis peu, face au souci qu'elle se faisait.

L'ombre de la mère, de la jouissance de la mère, n'est-elle pas ce qui vient s'interposer entre cette jeune femme et sa fille, dont la présence renvoie Anabelle à un Che vuoi trop angoissant?

Nous faisons ainsi l'hypothèse que l'opacité de la jouissance maternelle peut maintenir une femme dans une fixation mortifère à la mère, la renvoyant à réinterroger toujours, non sans angoisse, sa place dans le désir de l'Autre.

\section{Retour sur la question: ravage, jouissance féminine et maternel en tension}

La maternité ne relève pas davantage de l'instinct que du biologique pour l'être parlant en prise avec le désir de l'autre. Le vécu d'étrangeté devant l'enfant qui arrive peut être au premier plan notamment quand le manque d'amour de la mère, mais aussi du désir du père, a présidé à l'existence du sujet confronté à la maternité.

La clinique montre que celles dont le désir porte vers la maternité ont à se débrouiller avec des fantasmes dont le décryptage relève d'un vrai travail, possible sous transfert d'où peut se révéler parfois, la fertilité de l'inconscient.

Pas d'autre possibilité en effet que d'aborder l'enfant, et la maternité, autrement que par le biais de son fantasme, cela dans le meilleur des cas puisque, la précarité de certains montages que nous pouvons observer ne nous permettent peut-être pas de parler de fantasme, lequel se définit comme un scénario fixe permettant de réguler le rapport au monde et aux autres.

A partir de ces considérations, comment interroger la complexité de cette division entre mère et femme, et plus précisément la différence entre un ravage articulé au féminin et un ravage articulé au maternel?

A partir du Séminaire XX de Lacan, Sophie Marret-Maleval (2010) déduit que "la maternité est une suppléance au pas-tout". Lacan énonce en effet que là où la femme est "absente d'elle-même, absente en tant que sujet" car pas-toute dans la fonction phallique, "elle trouvera le bouchon de ce $a$ que sera son enfant" (Lacan, 1972-73), situant ainsi la mère du côté tout-phallique par opposition au pas-tout féminin. Ce qui signifie que l'enfant n'est pas simplement en position de phallus maternel mais qu'en place d'objet petit $a$, il "entre dans un rapport spécifique au pas-tout maternel" (Marret-Maleval (2010). 
Cette proposition nous permet de revenir sur cette expression "toute mère" qu'il s'agit de préciser: "si la femme n'est toute qu'en tant que mère, elle n'est pas non plus toute mère puisque son désir est loin d'être tout entier saturé par le signifiant" explique M. H. Brousse (2002, apud Marret-Maleval, 2010). Ceci nous laisse entrevoir comment l'enfant est pris dans le ravage maternel — si nous pouvons le nommer ainsi —, lui-même articulé à la jouissance féminine, et dévoilant "la part réelle de la relation de la mère à l'enfant" (Brousse, 2002, apud Marret-Maleval, 2010).

Outre le fait que cette partition mère/femme ne relève pas de l'évidence, comme le signale cette expression de "pas-tout maternel" et non plus seulement "féminin", cela ouvre une perspective à considérer dans la clinique: les fantasmes de maternité peuvent trouver à se décliner à plusieurs niveaux, au-delà de la dimension phallique de l'affaire que nous avons déployée à la lumières des écrits freudiens, puisque les mères, pour l'instant du moins, sont aussi toujours des femmes. Par conséquent, cette dimension d'illimité propre à la jouissance féminine peut tout aussi bien concerner une femme dans son rapport à un homme que dans son rapport à son enfant.

Ainsi, cette jouissance peut-elle se porter vers l'enfant quand il s'agit de combler ce pas-tout et de pallier à tout prix ce défaut d'être, d'une certaine manière pour refuser sa propre invention féminine, comme un large pan de la clinique le déploie.

$\mathrm{Ne}$ voit-on pas alors ainsi comment la mère peut "contaminer la femme" (Lacan, 1973b) dans son devenir mère?

Mater certissima dit le vieil adage de droit romain. Si la mère relève de la certitude, celle qui enfante a pourtant à devenir mère, tout comme il s'agit de devenir femme. Car à part le réel de la chair, quelle autre certitude peut-on poser quant à l'être mère ?

Le ravage, dans ses diverses figures, se décline dans le rapport d'une fille à sa mère (Lacan, 1973a), et d'une femme à son partenaire (Lacan, 1975-76, p. 101) là où la féminité est articulée à l'amour et à la jouissance, où une femme attend de la subsistance, terme que Lacan choisit pour définir le ravage et qui renvoie à l'existence.

Mais quand la mère contamine la maternité, "l'être mère" d'une femme — en l'occurrence sa fille — nous trouvons, sans doute aussi, la marque de cette "fixation" repérée par Freud alors qu'il signalait l'importance de la relation pré-oedipienne laissant tant de traces chez une femme (Freud, 1933/1984, p. 160). 
En regard de la dimension phallique, si fréquente dans la clinique (consistant à faire un enfant à sa mère par exemple) et ses diverses figures du ravage s'articulant au don, à l'amour et à l'exclusivité, nous devons pointer cette dimension de fascination et de fixation à la mère qui vient marquer une femme dans sa maternité. Là, se découvrent alors une identification pétrifiante à la mère, ou bien une sidération face à son énigmatique jouissance restée sans réponse comme nous l'avons décrit chez Anabelle, mais aussi dans les cas où la mère est atteinte dans son corps par la maladie, la marque, la cicatrice (Cf. Choukroun \& Ham, 2009/2).

Après Lacan, nous avons donc encore de quoi questionner comment la "Mère reste contaminer la femme pour le petit d'homme" (Lacan, 1973) chez le sujet masculin assurément, mais aussi côté femme, comment, à partir de la jouissance maternelle et du rapport de chaque sujet à l'Autre maternel, la Mère vient contaminer la femme dans sa féminité d'une part et dans son "être mère" d'autre part.

En somme, pas plus que l'être femme, l'être mère n'est donné d'emblée.

\section{Références}

Brousse, M.-H. (2002). Une difficulté dans l'analyse des femmes: le ravage du rapport à la mère, Ornicar?, 50, 93-105.

Choukroun, J., \& Ham, M. (2009). L'anorexie à l'adolescence: un paradigme du ravage au féminin? Cliniques méditerranéennes, 80, 249-265.

Freud, S. (1984). La féminité. Nouvelles conférences d'introduction à la psychanalyse. Collection Folio, Essais. Paris, FR: Gallimard. (Oeuvre originale publiée en 1933).

Freud, S. (1997). La vie sexuelle. Bibliothèque de Psychanalyse. Paris, FR: PUF. (Oeuvre originale publiée en 1907).

Lacan, J. (1957-58). Le séminaire. Livre V. Les formations de l'inconscient, Paris, FR: Editions du Seuil.

Lacan, J. (1961-62). Le séminaire. Livre IX. L’identification. Inédit.

Lacan, J. (1970-71). Le séminaire. Livre XVIII. D'un discours qui ne serait pas du semblant. Paris, FR: Editions du Seuil.

Lacan, J. (1972-73). Le séminaire. Livre XX. Encore. Paris, FR: Editions du Seuil.

Lacan, J. (1973a). L'étourdit, Scilicet 4, 21.

Lacan, J. (1973b). Télévision. Paris, FR: Editions du Seuil. 


\section{ARTIGOS}

Lacan, J. (1975-76). Le séminaire. Livre XXIII. Le sinthome. Paris, FR: Editions du Seuil.

Lacan, J. (1981). Le séminaire. Livre III. Les psychoses. Champ Freudien. Paris, FR: Editions du Seuil. (Oeuvre originale publiée en 1955-56).

Marret-Maleval, S. (2010). Le pas-tout sans le ravage, Conférence Antenne clinique de Dijon, Uforca, 4 décembre 2010.

Miller, J.-A. (1999). Un répartitoire sexuel, L'orientation lacanienne, Revue de La Cause Freudienne, 40, Maladies d'amour, Diffusion Navarin Seuil.

Solano Suarez, E, (mai 2003). Douleur de la féminité: entre l'être et l'existence, Trames actualité de la psychanalyse, Traversées du ravage entre mère et fille, De la férocité du contemporain, 32/33, pp. 31-45.

\section{Resumos}

(O gozo da mulher e a maternidade em questão)

A autora propõe confrontar os dois campos contíguos do feminino e do maternal das considerações freudianas sobre o "continente negro" que é a feminilidade e da noção de gozo feminino desdobrada por Jacques Lacan. À luz da psicopatologia psicanalítica clínica, este artigo quer salientar que, se o Édipo não faz A Mulher, o fato da devastação que é sua consequência, invade tanto o feminino como o materno. De fato, a parte opaca do prazer da mãe, ou seja, o feminino na mãe, contamina a feminilidade tanto quanto a maternidade de uma mulher, como demonstra a relação devastadora que pode existir entre uma filha e sua mãe.

A questão será, assim, de salientar que, não mais do que tornar-se mulher, tornar-se mãe não é dado desde o início, mas é construído a partir da relação com a linguagem, a fantasia, o gozo e o Outro na figura do amor materno, para cada uma.

Palavras-chave: Feminino, maternidade, gozo, devastação

(Feminine jouissance and the maternal in question)

The author proposes to confront the two contiguous fields of the feminine and the maternal based on Freud's considerations on the "black continent", i.e. femininity, and on the notion of feminine jouissance deployed by Jacques Lacan.

In the light of clinical psychoanalytical psychopathology, this article aims to highlight that if Oedipus does not make The Woman, the fact of the devastation which is its consequence invades the feminine, as well as the maternal. Indeed, the opaque part of the mother's jouissance, i.e. the feminine in the mother, contaminates both the woman's femininity and her maternity, as shown by the devastating relationship that can exist between a daughter and her mother. 
Thus, we point out that no more than becoming a woman, becoming a mother is not given from the outset, it is rather constructed from the relationship with language, with fantasy, with jouissance and with the Other in the figure of maternal love, for each one.

Key words: Feminine, maternal, jouissance, devastation

(El goce femenino y la maternidad en cuestión)

La autora propone confrontar los dos campos contiguos de lo femenino y lo materno, de las consideraciones freudianas sobre el "continente negro" que es la feminidad, y de la noción de goce femenino desarrollada por Jacques Lacan. A la luz de la psicopatología clínica psicoanalítica, este artículo quiere apuntar que, si el Edipo no hace La Mujer, el hecho de la devastación que es su consecuencia, invade tanto lo femenino como lo materno. De hecho, la parte opaca del goce de la madre, es decir, lo femenino en la madre, contamina la feminidad tanto como la maternidad de una mujer, como lo demuestra la devastadora relación que puede existir entre una hija y su madre. Se tratará, pues, de resaltar que, no más que el hecho de convertirse en mujer, el hecho de convertirse en madre no se da desde el principio, sino que se construye a partir de la relación con el lenguaje, la fantasía, el goce y el Otro en la figura del amor materno, para cada una.

Palabras clave: Mujer, maternidad, goce, devastación

Citação/Citation: Choukroun-Schenowitz, J. (2021, setembro). La jouissance féminine et le maternel en questions. Revista Latinoamericana de Psicopatologia Fundamental, 24(4), 570-585. http://dx.doi.org/10.1590/1415-4714.2021v24n4p570.5

Editora/Editor: Profa. Dra. Sonia Leite

Submetido/Submitted: 31.10.2020/10.31.2020 Aceito/Acepted: 15.3.2021/3.15.2021

Copyright: (C) 2009 Associação Universitária de Pesquisa em Psicopatologia Fundamental/ University Association for Research in Fundamental Psychopathology. Este é um artigo de livre acesso, que permite uso irrestrito, distribuição e reprodução em qualquer meio, desde que o autor e a fonte sejam citados / This is an open-access article, which permits unrestricted use, distribution, and reproduction in any medium, provided the original authors and sources are credited. 


\section{ARTIGOS}

Financiamento/Funding: Este trabalho não recebeu apoio. / This work received no funding.

Conflito de interesses/Conflict of interest: A autora declara que não há conflito de interesses. / The author declares that there is no conflict of interest.

Jessica Choukroun-Schenowitz

Psychologue clinicienne, BAPU Nice (Nice, France); Docteur en psychologie clinique

Maître de Conférences en psychopathologie clinique - Université Côte d'Azur - Campus

Carlone (Nice, France)

98 bd. Edouard Herriot

06209 Nice Cedex 3

jesschoukroun@yahoo.fr et Jessica.choukroun@univ-cotedazur.fr

https://orcid.org/0000-0002-3839-168X

This is an open-access article, which permits unrestricted use, distribution, 